\title{
Real-time Error Control Research Over Internet Communications
}

\author{
Wang Chunping ${ }^{1, *}$ and Chen Keming ${ }^{2}$
}

\author{
${ }^{I}$ School of Mathematics and Computer Science, XinYu University, JiangXi, 338004, China \\ ${ }^{2}$ School of Continuing Education, XinYu University, JiangXi, 338004, China
}

\begin{abstract}
With the development of computer technology and Internet technology, more and more real-time error control principles are implemented. Based on research of error control technology, In this thesis, we implement data packet's coding and decoding based on Reed-Solomon code through looking chart; and emulate the real-time error control systems that use error control methods of FEC and FEC/ARQ. Experiments results prove that coding and decoding based on ReedSolomon code through looking chart can improve the efficiency of coding and decoding, and experiments results also validate the FEC and FEC/ ARQ error control methods, that are based on the Reed-Solomon code, have fine characteristics and fine real-time, and decrease the code error ratio by $5 \%$ at least, at the same time, develop the data reliability and satisfy the QoS constraints.
\end{abstract}

Keywords: Code Error Ratio, FEC (Forward Error Correction), FEC/ARQ (Forward Error Correction/Automatic Repeat Request), Real-Time Error Control, Rs(Reed-Solomon) Code.

\section{INTRODUCTION}

Real-time Control System (RCS) is a reference model architecture, suitable for many software-intensive, real-time control problem domains. RCS is a reference model architecture that defines the types of functions that are required in a real-time intelligent control system, and how these functions are related to each other. RCS-3 produces a layered graph of processing nodes, each of which contains a task decomposition (TD), world modeling (WM), and sensory processing (SP) module. These modules are richly interconnected to each other by a communication system. RCS is neither a system design, nor a specification of how to implement specific systems. RCS prescribes a hierarchical control model based on a set of well-founded engineering principles to organize system complexity. All the control nodes at all levels share a generic node model.

With the continuous development of wireless sensor networks and a variety of emerging applications, wireless sensor networks for data transmission reliability requirements are important; thus the wireless sensor network error control study is particularly important. Wireless sensor nodes constrained by their own scale, i.e. they are energy limited. Therefore, if to give a large number of nodes in the network, an energy supplement is not feasible, which considers how energy limited circumstances, in order to minimize the energy consumption of nodes, extend the wireless sensor network's continuous working time. Error control in wireless sensor network research must also be based on reducing the energy consumption of nodes to ensure network stability and effective functioning. In wireless sensor networks, improving energy efficiency of each node is an excellent design of

*Address correspondence to this author at the School of Mathematics and Computer Science, XinYu University, JiangXi, 338004, China;

Tel: 13755586877; E-mail: 497496394@qq.com wireless sensor network primary consideration. Wireless sensor networks', in order to consume the least energy to provide reliable communication services, error control is based on the analysis of energy efficiency that becomes extremely important.

Most of the applications of media-stream communication need to transmit real-time data. We need to choose appropriate real-time error control code in order to get low code error ratio in the process of the communication. In this thesis, we raise an implementation of packet that is based on RS code, and emulate the real-time error control systems that use error control methods of FEC. The error control methods have a fine real-time, decrease the code error ratio, and develop the data reliability.

Real-time video and audio have limited tolerance for random loss within the compressed digital stream. The quality of decoded media at a receiver is subject to significant degradation as the result of excessive loss from network congestion or latency. In order to overcome the loss effects, error control techniques can be used. There have been three general error control approaches in the context of multicasting. In Retransmission- based Automatic Repeat request (ARQ), retransmissions occur only if data can be delivered before the real-time deadline. Two of such approaches are the error control aspect of LVMR presented by Li et al., [1] and STORM presented by $\mathrm{Xu}$ et al., [2]. In Forward Error Correction (FEC), the source assigns a portion of its bandwidth for proactive transmission of repair packets to the receivers. Among the rich set of articles in the literature, the two most closely related to our work are by Rubenstein et al., [3] in which the idea of using real-time reliable multicast using proactive FEC is proposed and Rhee et al., [4] in which a proactive FEC reliable multicast layering scheme is presented. There are also hybrid FEC-ARQ approaches suggesting different alternatives for proactive transmission of redundant packets based on retransmission requests. Chande et al., [5-6] and Chou et al., [7-9] proposed hybrid error con- 
trol systems by augmenting layered FEC with a pseudolayered ARQ system. Towsley et al., [10] and Nonnenmacher et al., [11] respectively analyzed the advantages of hybrid approaches over a stand-alone ARQ and in conjunction with local recovery.

\section{THE IMPLEMENTATION OF REAL-TIME ERROR CONTROL}

When the node and the communication distance are small, the channel has better conditions, an error occurs during data transfer with very small probability. It is no need to use an error correction encoding code, whose encoded energy consumption is far greater than the retransmission. ARQ error control method in this environment, has the highest energy efficiency. With the increase in the distance between nodes, FEC error control scheme advantage is reflected out. That is because the communication signal to noise ratio decreases with the increasing distance, and the receiver bit error rate is rapidly increased, so FEC protection scheme due to an error correction code, is declined at a slower pace. With the communication distance increase again HARQ scheme reflects the energy efficiency advantages; therefore, energy efficiency becomes a better solution. Comprehensive analysis of error control in wireless sensor networks by using different error-correcting codes and retransmission strategies, gives out various methods having advantages and disadvantages. However, because the wireless sensor network error control methods must consider energy efficiency, which will be inevitably and inextricably linked to node communication distance, wireless sensor networks must be able to independently determine the distance again to choose the best energy efficient error control methods.

Error control mode is used for correcting an error generated in the communication network and recovering the lost data. It is a technique to improve the reliability of network communication in a computer network and also a communication system for errors transmission and packing loss which may be generated in the process. It is the basic method of control which can be divided into four categories: forward error correction (FEC), automatic repeat request (ARQ), hybrid error control methods FEC / ARQ, information repeat request (IRQ) mode. The four methods have their advantages and disadvantages for different computer networks to achieve brief article below FEC and FEC / ARQ error control in two ways:

1) In FEC error control, redundant data is generated by encoding and the data transmission can recover the lost data in an error control mode. In this way, the sender sends a code capable of error correction and receives terminal after receiving the codes which is automatically corrected by the error correction decoder. The error occurs during transmission in this way and does not require a feedback channel in FEC error control mode. There are two methods to generate redundant data packet: media specific forward error correction (media-specific FEC); media-independent forward error correction (media-independent FEC). In this paper, the former media unrelated to correction mode is chosen.

2) FEC / ARQ error control method can combine the FEC and ARQ error control. FEC does not offer complete reliability of data, when used in combination. ARQ, FEC can produce more reliable than data transfer mechanism itself, which has a high efficiency in accordance with a combination of both correction mode. It can be divided into two types: hybrid ARQ feedback type I (ARQ type I) and hybrid ARQ feedback type II.

In computer networks, random errors and burst errors are often coexist. If a single random error correction code or correct burst error code cannot get good results, then you need to use both simultaneously correct error code which RS codes are the most widely used in this kind of code. It has a strong error correction capability. RS error correction code is recommended as a payload type of RTP.

In Markov channel model, the more commonly used models are Gilbert channel model, Bernoulli channel model, general Markov channel model and extended Gilbert channel model. In this paper, we use the Gilbert channel model because of its accuracy.

In Gilbert channel model, an alternative description can be based on a property of the model, that it generates a permutation of the initial deck in which each card is equally likely to have come from the first or the second packet. To generate a random permutation according to this model, begin by flipping a fair coin $n$ times, to determine for each position of the shuffled deck whether it comes from the first packet or the second packet. Then split into two packets whose sizes are the number of tails and the number of heads flipped, and use the same coin flip sequence to determine from which packet to pull each card of the shuffled deck.

Network under packet loss conditions can be modeled as two states: " 0 " indicates the normal destination status, which means a non-loss state; "1" indicates the status of packet loss. Gilbert packet loss model is shown in Fig. (1).

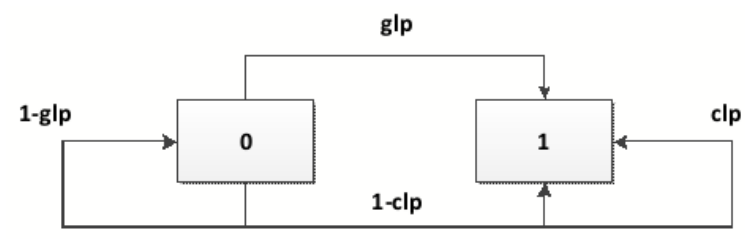

Fig. (1). The Gilbert packet loss model.

In Gilbert loss model, the following parameters are used:

(1) Conditional loss probability (CLP): the conditional loss probability is defined as under the packet $\mathrm{n}$ loss condition, the probability for the losing of $(n+1)$ lost packet.

(2) Unconditional loss probability (ULP): unconditional loss probability is defined as the loss probability of data packets $n$. It is expressed as the share ratio of the number of packets lost to the total number of packets sent.

(3) Good loss probability (GLP): good loss probability is defined as the probability for the loss of the next data packet under the condition of the former packet has lost.

The relationship between these three parameters can be expressed in the following equation:

$g l p=\frac{u l p(1-c l p)}{1-u l p}$ 
The Gilbert channel model is used to simulate the realtime data communication packet loss during transmission process. The implementation steps are as follows:

(1) Initialization channel state and packet loss rate;

(2) Definition clp and ulp parameter values;

(3) Calculating glp value according to the equation (1);

(4) Generate random packet loss rate in the $[0,1]$ range;

(5) Determine the current status of the channel is or is not 0 . If 0 , execute (6); otherwise execute (7);

(6) Random packet loss rate is compared with glp packet loss rate. If the packet loss rate is greater than the random glp, the loss rate of random packet loss rate is determined to be 0 , otherwise, go (8);

(7) Random packet loss rate is compared to the clp packet loss rate. IF the packet loss rate is greater than if the random clp, the loss rate of random packet loss rate is determined to be 0 , otherwise, go (8);

(8) Determine packet loss rate is or is not zero, if it is 0 , then execute (9); otherwise perform (10);

(9) The current packet and the channel state plus one, then go (4);

(10) The current packet is given up, and the channel state is set to 0 , go (4);

In real-time error control system illustrated in this paper, the packet loss process is simulated by the sender in Gilbert model.

When the FEC method is operated in a packet switched network transport layer or application layer, the data unit is the packet. In this paper, the RS code is used to the lookup table which is completed by polynomial multiplication and to achieve FEC encoded packet, the decoder or recover lost data packets. FEC coded data transmission to the group transport group (TG) is used as a unit, which includes k source packets and $h$ redundant packets. In order to improve the operation efficiency, before the encoding operation, the packet segmentation data is done.

In this paper, we define the word length of dividing unit which is noted as $\omega$ is 8 bit. Suppose in a transport group, the $\mathrm{j}^{\text {th }}$ binary word of the $\mathrm{k}^{\text {th }}$ source packet is $d^{j}$ and the $\mathrm{j}^{\text {th }}$ binary word of the $\mathrm{h}^{\text {th }}$ redundant packet is $r^{j}=\left\{r_{i}^{j}\right\}(i=1,2, \ldots, h)$. RS code encoding specific process steps are as follows:

(1) The $\mathrm{k}$ source packets are divided in the packet and then the $\mathrm{j}^{\text {th }}$ binary word can be obtained from $\mathrm{k}$ source packets, which can be expressed in the following equations:

$\overline{d^{j}}=\left\{d_{i}^{j}\right\}, \quad(i=1,2, \ldots ., k)$

(2) The polynomial element in Galois zone which is generated by $d^{j}$ binary word can be expressed by the following equations:

$\overline{d^{j}} \rightarrow d^{g f}=\left\{d_{i}^{g f}\right\}, \quad(i=1,2, \ldots ., k)$

(3) The RS transform in Galois zone can be get by the following equations:

$$
\overline{r^{g f}}=\left\{r_{i}^{g f}\right\}=R S\left(d^{g f}\right), \quad(i=1,2, \ldots ., h)
$$

(4) The $\mathrm{j}^{\text {th }}$ binary word of the $\mathrm{h}^{\text {th }}$ redundant packet can be got from the inverse mapping of $r^{g f}$ by the following equations:

$\overline{r^{g f}}=\left\{r_{i}^{g f}\right\}=R S\left(d^{g f}\right), \quad(i=1,2, \ldots ., h)$

(5) After the coding process of encoded word in all source packets, we do the combinations to get $\mathrm{h}$ redundant packets;

(6) The $\mathrm{k}$ source packets and $\mathrm{h}$ redundant packets are combined to form a transmission group TG.

In RS coding or decoding process, the basic polynomial which is shown in following is used.

$q(x)=x^{8}+x^{4}+x^{3}+x^{2}+1$

Galois zone is actually the extension zone of $G F(2)$, that is the $G F(2)^{8}$. The generating of mapping table is in fact the inverse process of the generating process of the elements in $G F(2)^{8}$ field. The Table I show the value of mapping and inverse mapping when $\omega=8$.

Table I. The value of mapping and inverse mapping when $\omega=8$.

\begin{tabular}{|c|c|c|}
\hline I & Mapping value & $\begin{array}{c}\text { Inverse mapping } \\
\text { value }\end{array}$ \\
\hline \hline 0 & -1 & 1 \\
\hline 1 & 0 & 2 \\
\hline 2 & 1 & 4 \\
\hline 3 & 25 & 8 \\
\hline 4 & 2 & 16 \\
\hline$\ldots$ & $\ldots$ & $\cdots$ \\
\hline $\mathbf{2 5 5}$ & 175 & 0 \\
\hline
\end{tabular}

Real-time FEC error control system is shown in Fig. (2). The system uses a client/server model.

\section{(1) Client communications}

In the client, the original data is used to obtain the stream pack, and an encoder for encoding the original data packet is got on the basis of FEC packet which is used to generate a redundant packet. The original packets constitute a coding block to form a transmission group TG through the network data transmission.

\section{(2) Server communication realization}

On the server side, the received data lost some packets through the network block. FEC decoder decodes the data packets within the error correction range. Code error correction capability is in limited scope and recovers lost data packets in the network to get the source of the send raw data streams. 


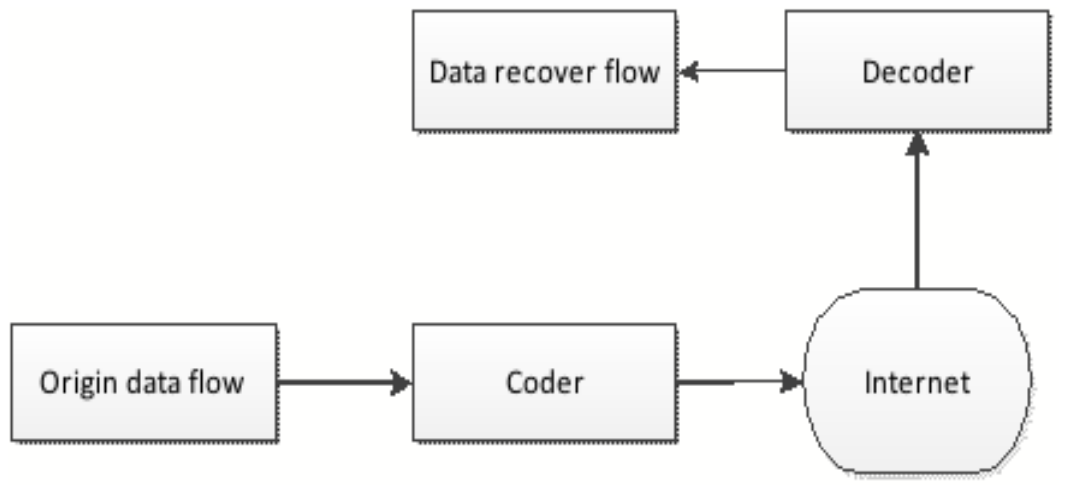

Fig. (2). The framework of real-time FEC error control system.

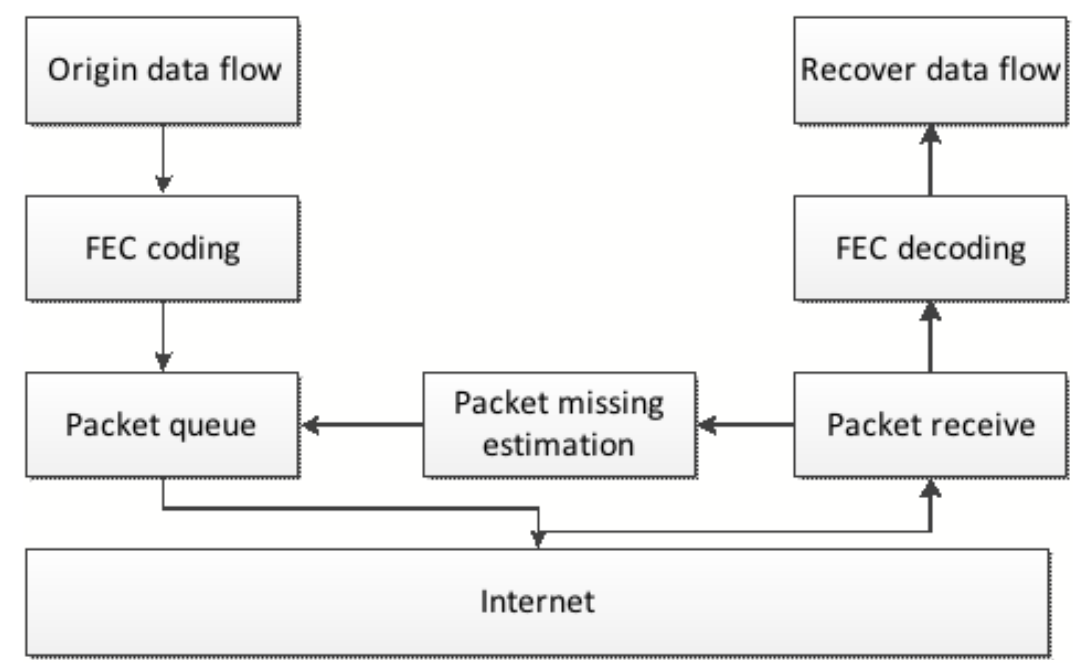

Fig. (3). The FEC/ARQ real-time error control system.

The receiving end opens multiple buffers for buffering the received data packet. The transmitting end keeps in order to avoid too fast sending rate which will bring the problem that the receiving end is too late to receive the packet which will cause the data loss.

The framework of FEC / ARQ real-time error control system is shown in Fig. (3). FEC error control is on the basis of real-time simulation system. This paper has realized the FEC / ARQ error control real-time simulation systems. The system also uses a client / server model.

\section{(1) Client communications}

In the client, the original data stream is packed, and an encoder for encoding the original data packet FEC RS code, to generate redundant packets. The original data packet is combined with redundant packets to form a coded block. Coded blocks are converged to form a transmission group TG through the network data transmission.

After receiving the ARQ retransmission request, based on the ARQ feedback information, the system immediately retransmit the lost packets. After completion of the retransmission, the system continues to send data frames.

(2) Server communication realization

Due to network congestion status, routing state of instability and other reasons, it will make some loss in data packet in the flow of packets sent by the client. On the server side, when receiving the lost packet stream, the system will calculate the number of packets lost in transmission group. If the number of lost packets has reached the limited capabilities of correcting RS code, then discard the transmission group and send ARQ request to the client. But it will receive the subsequent transmission of group memory into a temporary buffer.

\section{EXPERIMENTAL RESULTS}

In this section, Fig. (4) shows the data lost after FEC decoding. Where the clp in Gilbert model is defined 0.1, the coding redundancy in RS code system is 0.5 and the source packet number is 4 .

As can be seen from figure 4, by FEC encoding method, packet loss rate can be reduced by more than $5 \%$.

Fig. (5) shows the data lost after FEC decoding. Where the clp in Gilbert model is defined 0.1, the coding redundancy in RS code system is 2 and the source packet number is 5 . Then we change coding redundancy in RS code system to 2 and the source packet number to 4 .

Fig. (6) shows the data lost after FEC decoding. Where the clp in Gilbert model is defined 0.1, the coding redundancy in RS code system is 0.5 and the source packet number is changing. 


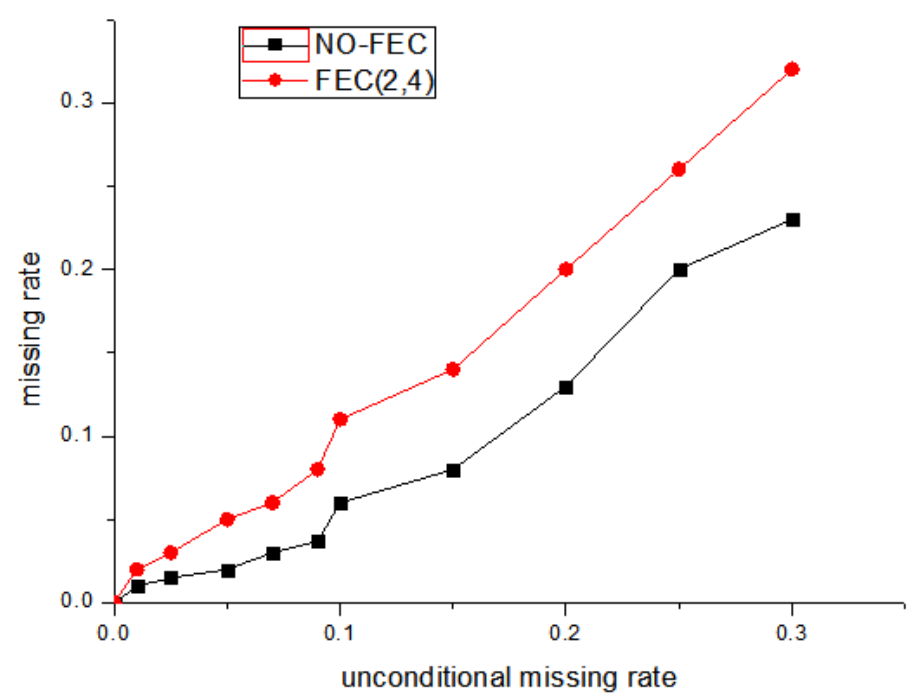

Fig. (4). The result of FEC (clp=0.1, $k=4, h / k=0.5)$.

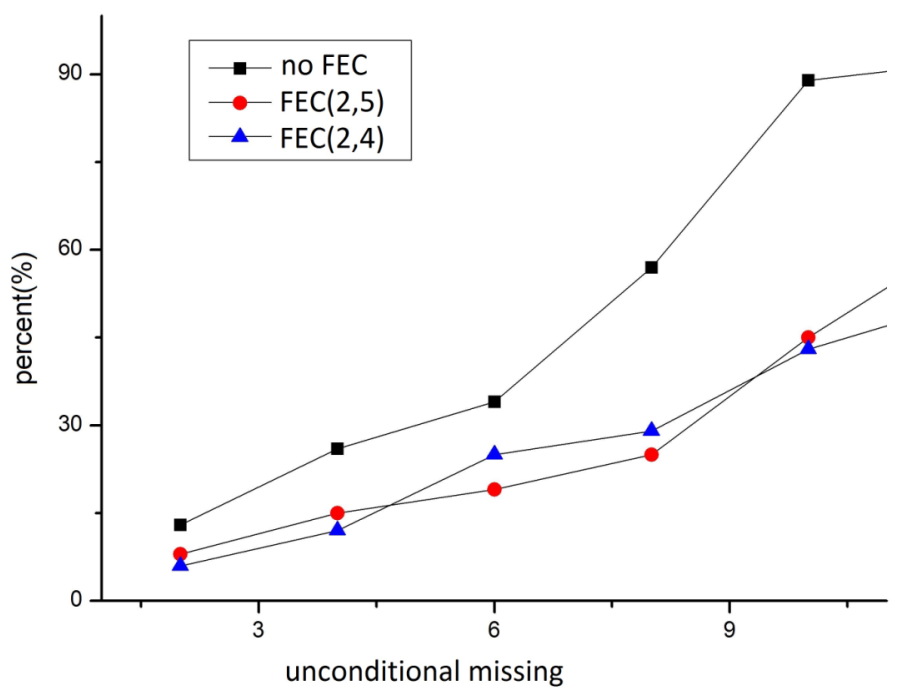

Fig. (5). The result of FEC $(\mathrm{clp}=0.1,(\mathrm{~h}, \mathrm{k})=(2,5),(2,4))$.

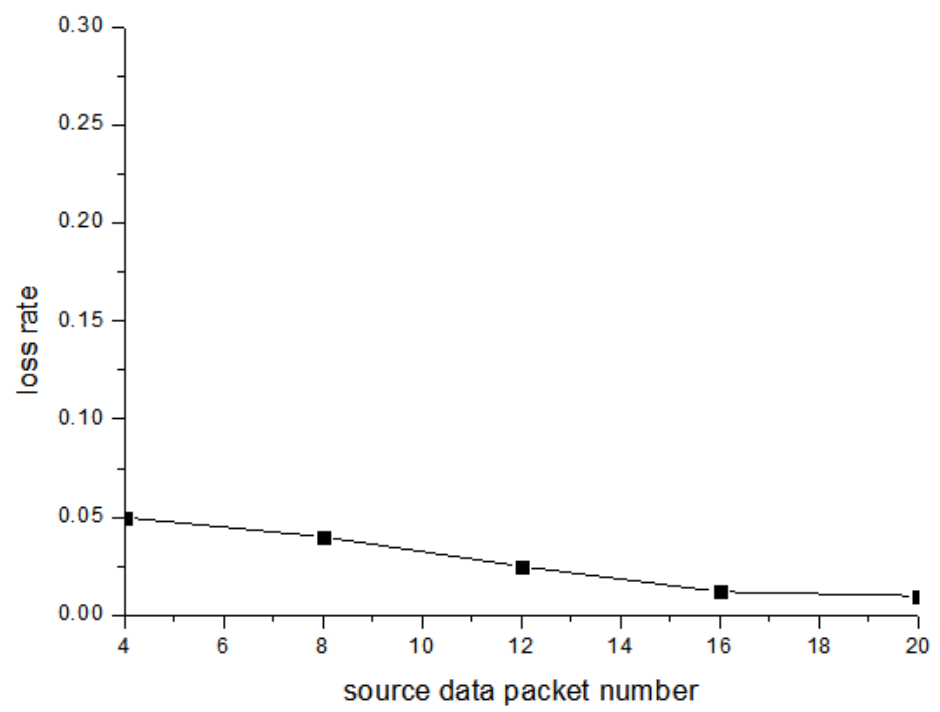

Fig. (6). The result of FEC ( $\mathrm{clp}=0.1, \mathrm{~h} / \mathrm{k}=0.5$, the source packet number is changing). 


\section{CONCLUSION}

In this paper, the RS code is combined with polynomial multiplication method using a lookup table. It effectively reduces the complexity of the polynomial operation and improves coding and decoding efficiency. It also reduces latency in the communication process. The results show that the proposed packet-based FEC encoding and decoding method for data processing unit show good performance in coding and decoding rate and can meet most of the requirements of the output rate of network applications. At the same time, it largely restores the lost data packets transmitted from the real-time simulation system. Based on the research of error control technology, In this thesis, we implement data packet's coding and decoding based on Reed-Solomon code through looking chart; and emulate the real-time error control systems that use error control methods of FEC and FEC/ ARQ. Experimental results prove that coding and decoding based on Reed-Solomon code through looking chart can improve the efficiency of coding and decoding. Our results also validate the FEC and FEC/ ARQ error control methods that are based on the Reed-Solomon code. They have fine characteristics and fine real-time, and can decrease the code error ratio at least by $5 \%$, besides developing the data reliability and satisfying the QoS constraints.

\section{CONFLICT OF INTEREST}

The authors confirm that this article content has no conflict of interest.

\section{ACKNOWLEDGEMENTS}

Declared none.

\section{REFERENCES}

[1] N. Shacham, and P. McKenney, "Packet Recovery in High Speed Networks Using Coding and Buffer Management," IEEE Computer and Communivation Societies, no. 1, pp: 124-131, 1990.

[2] P. Frossard, "FEC Performance in Multimedia Streaming," IEEE Communications Letters, vol.5, no. 3, pp. 12-22, 2001.

[3] P. Frossard, and O. Verscheure, "Joint Source/ FEC Rate Selection for Quality Optimal MPEG 22 Video Delivery," IEEE Transaction on Image Processing, vol. 10, no. 12, pp. 181-192, 2001.

[4] E. Biham, and A. Shamir, "A Differential Cryptanalysis of the Data Encryption Standard," Springer-Verlag, pp. 126-129, 1993.

[5] M. Matsui, "The first experimental cryptanalysis of the data encryption standard," In: Proceedings CRYPTO 94, Lecture Notes in Computer Science, no. 839, pp. 1-11, 1994.

[6] K. Xu, Y. Liu, and W. Liu, "The Design and Implementation of Security Access Proxy in Database application System," Computer Engineering and Application, no.1, pp. 105-107, 2001.

[7] J. Zhang, and Y. Dai, "Design and Implementation of Network Encrypted Database System Based on Proxy," Computer Engineering and Application, no. 18, pp. 196-198, 2002.

[8] E. Biham and A. Shamir, "A Differential Cryptanalysis of the Data Encryption Standard, Springer-Verlag, pp. 126-129, 1993.

[9] M. Matsui, "The first experimental cryptanalysis of the data encryption standard, In Yvo G. Desmedt, editor, In: Proceedings CRYPTO, Lecture Notes in Computer Science, no. 839, pp. 1-11, 1994.

[10] J. Rosenberg, and H. Schulzrinne, "An RTP Payload Format for Generic Forward Error Correction," IRTF Audio Video Transport $W G$, pp. 191-200, 1998.

[11] E. N. Gilbert, "Capacity of a Burst Noise Channel," Bell Syst Tech, vol.3, no.9, pp. 125-126, 1960.

\author{
Received: May 26, 2015 \\ Revised: July 14, 2015 \\ Accepted: August 10, 2015 \\ (C) Chunping and Keming; Licensee Bentham Open.
}

This is an open access article licensed under the terms of the (https://creativecommons.org/licenses/by/4.0/legalcode), which permits unrestricted, noncommercial use, distribution and reproduction in any medium, provided the work is properly cited. 